\author{
Military Technical College \\ Kobry El-Kobbah, \\ Cairo, Egypt.
}

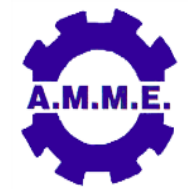

\author{
$18^{\text {th }}$ International Conference \\ on Applied Mechanics and \\ Mechanical Engineering.
}

\title{
DEVELOPMENT OF A NUMERICAL TOOL AND APPLICATION OF A NOVEL NATURAL FIBRE MATERIAL FOR THE REDUCTION OF VEHICLE CABIN NOISE
}

\author{
D. A. Desai ${ }^{1}$, R. K. Dunne ${ }^{2}$ and R. Sadiku ${ }^{3}$
}

\begin{abstract}
The level and quality of noise experienced by occupants in transportation systems such as military vehicles are issues of increasing concern and are among the major challenges of the automotive industry apart from reducing vehicle mass and product recyclability. Researches have shown that the vibration behaviour of panels that enclose the passenger cabin can affect low-frequency interior noise resulting in adverse effects on the human body or vehicle occupants. However, a compelling amount of anecdotal evidence reported that the characterisation of structure-borne panel vibration and its subsequent noise emission characteristics employing recyclable, sound absorbing, natural fibre composites with an Acrylonitrile Butadiene Styrene (ABS) matrix has not yet been studied within a vibro-acoustic setting. Thus, the primary contribution of this original work was to implement a novel poroelastic natural fibre damping material into a flexible numerical model to predict and improve the vibro-acoustic performance of a vehicle's cabin when provoked by an external structural forcing function. With reference to an undamped vehicle, a discrete, frequency domain numerical model is developed and experimentally validated based on frequency response data. It was observed that the proposed numerical model effectively and efficiently simulates the noise levels within the vehicle model while the newly developed natural fibre material does reduce cabin noise to a perceivable degree.
\end{abstract}

\section{KEY WORDS}

Vibration, Automotive interior noise, Finite element analysis, Fluid-structure interaction, NVH.

1 Doctor, Dept. of Mechanical Engineering, Faculty of Engineering and the Built Environment, Tshwane University of Technology, South Africa.

2 Graduate student, Dept. of Mechanical Engineering, Faculty of Engineering and the Built Environment, Tshwane University of Technology, South Africa.

3 Professor, Dept. of Chemical, Metallurgical and Material Engineering, Faculty of Engineering and the Built Environment, Tshwane University of Technology, South Africa. 


\section{INTRODUCTION}

The reduction of interior noise in transportation systems is an important concern in noise, vibration, and harshness (NVH) design for various reasons and has significant advantages in military applications. Furthermore, the noise signatures of the passenger cabin contribute substantially towards passenger comfort and the overall impression of the vehicle quality which in turn has a great influence on the buying decision of the customer. Because the time to market is constantly decreasing while the pressure to save costs is increasing, NVH engineers seek to define and improve a vehicle's vibro-acoustic comfort characteristics as early as possible. For this reason, a predictive finite element based simulation model is developed in this work to study the acoustic performance of a passenger cabin in the low-frequency range (20 to $200 \mathrm{~Hz}$ ), as evidence from biodynamic studies demonstrate that the lowfrequency range is considered most important and troublesome in terms of its adverse effects on the human body [1].

Complimentary to this, the need for innovative recyclable and environmentallyfriendly material is of an ever increasing trend. This is due partly to concerns over rising costs, negative environmental impact and unstable supply of fossil fuels [2]. The automotive industry in particular is under ever increasing pressure from governing bodies. These governing bodies, such as the European Commission are pushing for revised legislation and regulations concerning the issue of product recycling, carbon dioxide $\left(\mathrm{CO}_{2}\right)$ emissions and protection of resources [3], [4]. One of the most promising efforts that can be made in reducing environmental pollution is through the use of natural fibre materials [5]. By using such materials in the production of products, a more recyclable and environmentally-friendly vehicle can be produced. Therefore, in the present study which is part of an already published work [6], the performance of a newly developed natural fibre composite is been investigated in the context of vehicle cabin noise reduction using a numerical model.

\section{DAMPING MATERIAL AND NUMERICAL MODEL DEVELOPMENT}

\section{Natural Fibre Damping Material}

According to the literature review, Kenaf and Sisal natural fibres possess a number of good properties, which are needed in the present work. Therefore, these fibres were selected as basic constituents for the development of the final composite, due to their low cost, low density, good toughness, recyclability, good sound absorption properties, favourable airflow resistivity and biodegradability [7-9]. The final composition of the newly developed poroelastic fibre contained a $70 \%$ of Sisal fibre and $30 \%$ of Kenaf fibre mix. The matrix used to bind the fibres was made from discarded 3D printing Acrylonitrile Butadiene Styrene (ABS) scrap. This matrix improved the recyclability and environmentally-friendly nature of the proposed composite. The material properties of the developed composite acquired by physical testing by the author are listed in Table 1 and the detailed development, manufacturing and characterization of this fibre is described in Dunne et al. [6]. This poroelastic natural fibre material was attached to the interior walls of the vehicle cabin (named as treated or trimmed surfaces) using a good quality ABS adhesive, and its effectiveness in terms of sound attenuation (damping) is investigated in this work. 


\section{Development of Numerical Vehicle Model}

The main objective of the present work is to develop a predictive tool, using finite element analysis (FEA) software capable of predicting efficiently and effectively the sound pressure levels (SPL's) within a vehicle cabin treated with a natural fibre composite. However, a well-developed, scaled-down FEA model has the advantage of predicting accurate resonance frequencies and SPLs at a much lower computational cost due to its size, hence the attractiveness of it. Furthermore, due to laboratory space restrictions and experimental manufacturing costs, a scaled-down vehicle model is developed and employed in this work. Furthermore, one of the fundamental structural design considerations for a body-in-white (BIW) vehicle is the overall dynamic behaviour in both torsion and bending [10]. Hence, the torsional and bending stiffness is of primary importance for dynamic similarity in the development of a scaled-down model. Subsequently, a stiff, one-fifth, scaled-down vehicle structure was constructed in ABAQUS finite element software using similitude theory in order to maintain approximate relative occurrence of acoustic and structural modes over the low-frequency domain on par with a typical full-size real BIW vehicle structure as shown in Fig. 1. According to typical service conditions, contact and damping phenomena have been considered so that the dominant physics and relevant phenomena can be adequately represented.

The dynamic similarity scaling laws used to govern the developed scaled vehicle model behaviour are as follows [11]:

$$
K_{m}=\frac{1}{N} K_{p}
$$

where $K_{m}$ is the stiffness of the scaled model, $N$ the scaling factor $(=5)$ and $K_{p}$ is the stiffness of the full scale vehicle and

$$
F_{m}=\frac{1}{N^{2}} F_{p}
$$

where $F_{m}$ is the force acting on the scaled model and $F_{p}$ is the force on the full scale vehicle.

According to Desai [1], the bending stiffness of a typical vehicle is in the range of about $1.5 \mathrm{kN} / \mathrm{mm}$. By applying Eqn. (1), the bending stiffness of the scaled model is in the range of $0.3 \mathrm{kN} / \mathrm{mm}$. According to Tebby et al. [10], the torsional stiffness target for a typical vehicle is in the range of $10-18 \mathrm{kN} . \mathrm{m} / \mathrm{rad}$. Eqn. (1) is also used to calculated the torsional stiffness of the scaled model which is in the range of $2.8 \mathrm{kN} . \mathrm{m} / \mathrm{rad}$. By careful design, the bending and torsional stiffness of the developed scaled model was numerically computed (finite element model) as $0.212 \mathrm{kN} / \mathrm{mm}$ and $2.207 \mathrm{kN} . \mathrm{m} / \mathrm{rad}$, respectively. However, it should be noted that due to the nature of varying vehicle stiffness available in practice (owing to different vehicle body structures in existence); many researchers report a range of varying values. Hence, the stiffness's numerically computed are in compliance to the above calculated values. Subsequently, a complete scaled simulation vehicle model (Fig. 1 clad with various body panels) was developed in ABAQUS as depicted in Fig. 2. 


\section{Modelling approach}

The modelling method employed consists of a fully-coupled (body structure to interior air volume) vibro-acoustic analysis in order to represent the dominant physics involved as described below. The complete finite element simulation has passed through the following four steps:

Step 1: $\quad$ Nonlinear static analysis.

Purpose: To include the effects of gravity on the system for improvement of modelling accuracy.

Step 2: $\quad$ Linear perturbation static analysis.

Purpose: To include the computation of residual modes for the purposes of increasing accuracy in subsequent mode-based procedures.

Step 3: $\quad$ Linear perturbation frequency analysis.

Purpose: $\quad$ To extract the coupled acoustic-structural Eigen modes of the system in order for the subsequent structure's response to steady-state harmonic excitation to be computed.

Step 4: $\quad$ Steady-state dynamic, mode-based analysis.

Purpose: To determine the vibro-acoustic response of the coupled system subjected to a multi-frequency harmonic excitation acting at an excitation point coinciding with a front wheel suspension location.

This final step, which employed modal techniques, was based on frequency response function (FRF) simulations of the fluid-structure interaction between the passenger cabin and its body structure with respect to the structural excitation.

The vehicle structure and its respective components were modelled as deformable bodies including the developed poroelastic fibre and flexible wall panels enclosing an acoustic cavity. Since testing revealed that the natural frequencies for both the structure and acoustic medium span the same range, a coupled approach was adopted as these "vibro-acoustic" modes provide the information necessary to understand the physical phenomena under investigation. The discretized equations of motion for a poroelastic material attached to a flexible structure can be written in matrix form as [12]:

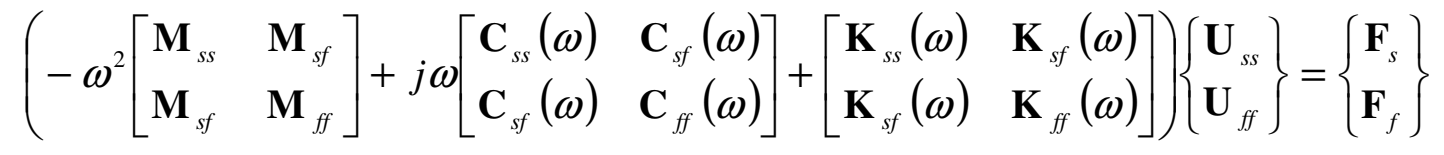

where $\mathbf{M}, \mathbf{C}$ and $\mathbf{K}$ are the equivalent mass, damping and stiffness matrices and the subscripts, ss, ff and sf denote solid phase, fluid phase and solid-fluid coupled phases inside the poroelastic material, respectively. $\mathbf{U}_{s s}$ is the solid phase displacement and $\mathbf{U}_{f f}$ is the fluid phase displacement, respectively. Eqn. (3) is solved in ABAQUS finite element software using the modal superposition procedure. Since the acquisition of actual damping characteristics in complex structures is very difficult, an approximate spectral modal damping scheme is employed, which introduces an energy dissipation term $\delta W_{\text {diss }}$ in the form [1]:

$$
\delta W_{\text {diss }}=2 \zeta_{i} \omega_{i} \dot{u}_{i}
$$


where $\zeta_{i}$ is the ${ }_{i}{ }^{\text {th }}$ modal damping ratio, $\omega_{i}$ is the natural frequency and $\dot{u}_{i}$ is the velocity. It should be noted that the damping values used in Eqn. (4) were obtained experimentally by the author and were subsequently post processed using the Engineering Data Management (EDM) software package.

Finally, the A-weighted SPL response at a particular nodal location $L_{p}$, is expressed as [13]:

$$
L_{p}=20 \log _{10}\left[\frac{\bar{p}_{r m s}}{p_{r e f}}\right]+A \text { weighting }
$$

where according to [14]

$$
\bar{p}_{r m s} \approx \frac{p_{\max }}{\sqrt{2}}
$$

and $\bar{p}_{r m s}$ is the root mean square pressure, $p_{r e f}$ is the standard reference pressure which is equal to $2 \times 10^{-5} \mathrm{~Pa}$, and $p_{\max }$ is the peak acoustic pressure.

The above descriptions allowed for the simulation of the proposed model and the results of the predicted and measured SPL's are graphically represented in Figs. 3 and 4 , respectively. It can be seen from both figures that the numerical results closely correspond to the measured results. However, Figs. 3 and 4 only depict the representativeness of the purposed model, and do not give a realistic indication of the effect the natural fibre composite has on the internal SPL's. Therefore, an effort was made to simulate the proposed natural fibre composite in such a way that it would realistically represent the treated surfaces in a typical vehicle. This will allow for the generation of more useful data in terms of the attenuation in SPL's due to the application of the developed poroelastic natural fibre sound absorbing composite.

Hence, it is noted from the literature that up to $20 \mathrm{~kg}$ of textiles, carpets and other trim parts are used in the interior of the vehicle [15], and the target thickness for automotive lining is in the range of $11-12 \mathrm{~mm}$ [16]. Hence, scaling the thickness by a factor of 5 gives an approximate thickness of $2.5 \mathrm{~mm}$. However, the poroelastic natural fibre mass cannot simply be scaled and needs a scaling law which can be described as [11]:

$$
m_{\text {scaled }}=\frac{m_{\text {actual }}}{N^{3}}
$$

Therefore, taking the mass of fibre, for the carpet and headliner only, in a full vehicle to be approximately $14.5 \mathrm{~kg}$, since the total mass of all textiles, carpets and headliners are approximately $20 \mathrm{~kg}$, and substituting it into Eq. (7), a scaled fibre mass of $116 \mathrm{~g}$ is computed.

This translates to an internal cabin area coverage of $0.39576 \mathrm{~m}^{2}$ which is approximately $44 \%$ of the internal surface area.

This damping fibre material was modelled using impedance properties and meshed with first-order hexahedral, solid brick elements of type C3D8P in order to achieve high accuracy. The scaled poroelastic natural fibre trim can be seen in Fig 5. 


\section{Mesh design}

The structural members and panels of the vehicle frame were modelled in a 3D modelling space using a deformable type shell extrusion. Surface-to-surface tie constraints were used in order to tie all contact surfaces together so that there is no relative motion between them and this method provides more accurate results than node-to-surface tie constraints.

Meshing considerations in a dynamic structure in FEA are very important and special care was taken since the number of elements used to model a given structure is a compromise between solution accuracy and cost [1]. Although the meshes at the acoustic-structural tied boundary may be nodally nonconforming, mesh refinement, which depends on wave speeds in the two media, affects the accuracy of the solution. The mesh for the medium with the lower wave speed (air) should generally be more refined and therefore should be the slave surface. Since material properties affect mesh parameters for wave propagation problems and hence affect the accuracy of the solution, the discretization protocol of the finite element method require at least six nodes per wavelength. According to [17], 6 to 10 elements per wavelength (structural and acoustic) were used when discretising the parts in order to maintain a reasonable level of accuracy. Mesh convergence studies in the case under investigation revealed that the use of a mesh with size of $9 \mathrm{~mm}$ (for both structure and acoustic media) was ideal with very little loss of accuracy and a substantial saving on CPU time. However, this was done cautiously and all structural members were meshed with at least 10 elements per wavelength.

The cabin frame and panels were meshed with first-order, quadratic thin shell, reduced integration elements, of type S4R with hourglass control in order to reduce CPU memory requirements. The acoustic domain was discretized using threedimensional, first-order hexahedral, solid brick elements of type AC3D8 in order to achieve maximum accuracy. The porous sound absorbing material was discretized using, first-order hexahedral, solid brick elements of type C3D8P in order to achieve a high accuracy. The acetoxy silicone material, used to secure the front panel during testing (needed for cabin accessibility as described below), was discretized using, first-order hexahedral, solid brick elements of type C3D8IH. The total system model comprised a total of 313490 elements.

\section{Loading and boundary conditions}

The typical wheel suspension input forces of a Sedan vehicle, when driving on tarred road surfaces, are in the range of $100 \mathrm{~N}$ [18]. However, the continual changing of bending and torsional loading on the vehicle structure due to the irregular surface the vehicle transverses, contributes significantly to the vehicles structural vibration. Therefore, applying Eqn. (2) in order to preserve dynamic similarity, gives an input force of $4 \mathrm{~N}$. However, it should be noted that the force acting on the wheel is assumed harmonic in nature. Therefore, the input forcing function can be expressed as [19]:

$$
F_{y}=4 \sin \omega t
$$

where $\omega$ is the angular frequency and $t$ is the time. This loading was applied sinusoidally as a frequency sweep in the low frequency range of 20 to $200 \mathrm{~Hz}$ with increment of $0.25 \mathrm{~Hz}$.

The dynamics of the system were analysed under free-free boundary conditions. 


\section{EXPERIMENTAL CHARACTERIZATION AND SETUP}

In order to initially validate the proposed numerical model (to be used for subsequent analysis), an experimental modal analysis using the single reference method with shaker excitation was conducted to determine correlation of resonance frequencies between the numerical and experimental models. The test structure was manufactured and assembled in a similar way to that of an actual Sedan vehicle. All air gaps in the vehicle body were sealed with high-quality non-porous duct tape in order to acoustically seal the interior cavity, thus minimizing acoustic losses. The test vehicle was suspended using four soft elastic bands in an overhead fashion to simulate the free-free boundary conditions used in the dynamics calculations. Subsequently, a steady-state, forced response, harmonic analysis was carried out in order to measure the vibration of the vehicle cabin panels, in terms of a FRF, and the SPL's within the cabin. All equipment used was calibrated and operated according to the manufacturer's recommendations. A schematic representation of the used test setup is shown in Fig 6.

The procedures followed for the experimental modal analysis are as follows:

- The first step was suspending the scaled vehicle structure by four soft elastic cords. The cords were attached at the top front and back corners close to nodal points represented in numerical simulation. These elastic cords allowed for a close replication of free-free boundary conditions which were needed in modal analysis for accurate extraction of mode shapes.

- Thereafter, an electromagnetic shaker, Sentek Dynamic exciter model MS-1000 was attached to the experimental model via a stinger. A stinger was connected to a calibrated PCB force transducer with a sensitivity of $2.248 \mathrm{mV} / \mathrm{N}$, which was attached to the bottom panel of the vehicle coinciding to the front wheel excitation position. A force transducer connected to the stinger was attached to the vehicle's base using good quality cyanoacrylate adhesive as recommended by [20] which allowed for a rigid bond.

- The next step was the attachment of miniature DeltaTron accelerometers. Special consideration was taken since the mounting method of an accelerometer has an influence on the accelerometer's frequency response. The accelerometers were attached to the right side panel along its centre line as shown in Fig. 7. Since the panels to which the accelerometers were mounted, were very thin $(0.8 \mathrm{~mm})$, stud mounting was not practical [20]. Furthermore, the frequency range of excitation was low; hence the accelerometers were attached using high quality bees wax. The accelerometers were pressed onto the side panel in order to ensure the thickness of the wax between the side panel and the accelerometer was minimal. This was done in order to reduce any elastic effects the wax may exert when subjected to excitation. In this study, three accelerometers were used in order to ensure accuracy of the results and to capture the bending behaviour of the panel more accurately for subsequent analysis.

- Next the shaker was clamped down using G-clamps in order to absorb reaction forces, prevent movement of the shaker and to ensure only normal forces are transmitted to the constructed floor panel. 
- The force transducer and accelerometers where then connected to the signal converter and spectrum analyzer Model Coco-80.

- Since the specially developed poroelastic natural fibre composite was to be attached within the cabin, it was necessary that the front panel be detachable (accessible). Hence, the front panel was attached using an acetoxy silicone. This allowed for easy removal of the front panel as well as providing a fairly rigid, acoustically sealed cabin. The poroelastic natural fibre composites were attached to the inner bottom, top and rear surfaces of the vehicle cabin using a very thin layer of $A B S$ which was painted on the relative surfaces as can be partially seen in Fig. 8.

Lastly, a steady-state, forced response, harmonic analyses was conducted, the procedure of which is similar to the above modal analysis with the only difference being the replacement of the accelerometers with microphones as can be seen in Fig. 9. In order to minimise the possibility of contaminated data, tribo-electric and sensitivity effects due to cable whip were reduced by using stiff, graphite cables that were laid straight and taped down to the surface of the vehicle body with high-quality duct tape. Data cables were separated from power cables to reduce electromagnetic interference. The cables were also checked for sharp bends, cuts or any type of damage since this could cause erroneous measurements [21]. Relevant precautions were taken to minimise mounting resonances. Background noise checks using the $L_{e q}$ method where also performed before measuring the cabin SPL's in order to ascertain that the background noise floor had no effect on the measured results. The shaker was driven with a swept sine signal, which was band limited to the frequency range of interest coincident with that of the finite element model. The data was sampled at $512 \mathrm{~Hz}$ with a $0.25 \mathrm{~Hz}$ resolution corresponding to the numerical model.

\section{RESULTS AND DISCUSSION}

With a model size of approximately 1.27 million degrees-of-freedom (including contact elements and Lagrange multiplier variables), the model converged at 27.383 minutes of CPU time. The analyses were executed on a $3 \mathrm{GHz}$ i7 Intel ACER laptop with $4 \mathrm{~GB}$ of RAM, running on a Windows 7 platform. Fig.10 depicts the resonant frequency versus mode number for both the experimental and simulated models. The largest difference in natural frequencies between the numerical model and measured values were $4.21 \%$ at mode number 12 . It can be seen that the difference between the experimental and the predicted values are small and therefore prove the predictive capabilities of the model.

Furthermore, the experimentally-determined damping ratios varied between 0.022 and 0.263 and were subsequently used as input into Step 4 of the developed numerical model.

The relations between the experimental and predicted A-weighted SPL's at the front driver's head position and the right rear passenger's head position are plotted in Figs. 11 and 12, respectively. Moreover, Figs. 11 and 12 plot the relation between the SPL's of the trimmed and untrimmed numerical models at similar positions as that in Figs. 3 and 4, respectively. By analysing these figures, it can be seen that these data correlates relatively well over most the frequency range despite the complexity of the 
vehicle structure and the poroelastic natural fibre sound absorbing material employed.

Furthermore, based on the direct relationship between these figures and Table 2, it can be seen that there is an attenuation of more than $3 d B(A)$ at most resonance points at the front and rear head positions. This may be regarded as significant in the light of the current refinement levels of modern vehicle cabins notwithstanding that a $3 d B$ change in SPL is perceivable to the human ear [22]. Furthermore, greater levels of acoustic damping can be achieved by employing appropriate material distribution optimization algorithms.

The overall results indicate that the numerical model predicts the dominant resonance peaks as well as the general trend of the vibro-acoustic behaviour demonstrating the accuracy of the developed numerical model. Though a direct comparison to reality is not possible (due to the uniqueness of this investigation), it was also found that the measured and simulated resonance peaks correlated very well with that of a full sized vehicle as reported by Zheng et al. [23].

The small inaccuracies observed may be attributed to various factors such as imperfect boundary conditions (free-free boundary conditions) as well as the microphone connections through the top panel of the structure, interference within the measurement equipment, limitations in the physical measurement process such as dynamic interaction between vehicle structure and the shaker (normal forces not perfectly translated through the stinger into the vehicle structure), inaccuracies in the measurement of the poroelastic natural fibre materials, airflow resistivity which is used to calculate the permeability used in the numerical model as well as inaccuracies associated with the construction of the experimental model, to name a few.

A comprehensive analysis on the physical capability of the natural fibre's ability to reduce noise is discussed in [24-25].

\section{CONCLUSIONS}

In this work, a computational procedure with a numerical model, based on the finite element approach, has been presented in order to predict acoustic performance as a function of a newly developed poroelastic fibre damping material. The study showed that achieving a high degree of correlation for complex structures, such as the one under investigation, is difficult because simulation models generally represent an ideal discretized world, in contrast to experiments where variability is present. Despite this, the developed numerical model was successfully validated by actual measurements and was amenable to furnishing reliable frequency response information at the points of interest.

The study further validated the sound absorption ability of an alternative recyclable natural fibre product, which can be used for the attenuation of SPL's within a cabin enclosure. This is based on the fact that the study revealed an attenuation of more than $3 d B(A)$ at most resonance points at both the front and rear head positions. This reduction in noise levels is not of a superficial nature but is perceivable to the human 
ear, which is advantageous to numerous industries such as the military in stealth missions.

The insights gained into the noise generation and transmission behaviour of the system under investigation may be materialized into realistic design modifications enabling $\mathrm{NVH}$ engineers to move closer towards cabin acoustic refinement in pursuit of their quest for quieter passenger cabins.

\section{REFERENCES}

[1] D. Desai, "Prediction and Reduction of Low-Frequency Vibro-Acoustic Transmission Through Automotive Door Mounts", Thesis, Tshwane University of Technology, Pretoria (2010).

[2] T. Sen and H.N.J Reddy, "Various Industrial Applications of Hemp, Kenaf, Flax and Ramie Natural Fibres", International Journal of Innovation, Management and Technology, 2(3), pp.192-198 (2011).

[3] E. Ghassemieh, "New Trends and Developments in Automotive Industry", UK: InTech (2011).

[4] K. Pickering, "Properties and Performance of Natural-Fibre Composites", Great Abington (2008).

[5] R. Dunne, D. Desai, R. Sadiku, "A Review of Natural Fibres, Their Sustainability and Automotive Applications", Journal of Reinforced Plastics and Composites, 35(13), pp.1041-1050 (2016).

[6] R. Dunne, D. Desai, R. Sadiku, "Material characterization of Blended SisalKenaf Composites with an ABS Matrix", Applied Acoustics, 125(Supplement C), pp.184-193 (2017).

[7] M.J. Saad and I. Kamal, "Kenaf Core Particleboard and its Sound Absorbing Properties", Journal of Science and Technology,4(5), pp. 23-34 (2012).

[8] F. Asdrubali, "Survey on the Acoustical Properties of New Sustainable Materials for Noise Control", Proceedings of the Euronoise 2006 Conference, 30 May-1 June 2006,Tampere, Finland (2006).

[9] L.J. Azevedoa and M. Nabuco, "Sound Absorption of Sisal Fiber Panels", The 2005 Congress and Exposition on Noise Control Engineering, Rio de Janeiro, Brazil: Institute of Noise Control Engineering ( INCE ) (2005).

[10] S. Tebby, E. Esmailzadeh and A. Barari, "Methods to Determine Torsion Stiffness in an Automotive Chassis, Computer-Aided Design \& Applications", pp. 67-75 (2011).

[11] S. Chakraborty, "Mechanics Over Micro and Nano Scales", Springer, New York (2011).

[12] G.P. Mathur, C.L. Chin, M.A. Simpson and J.T. Lee, "Structural Acoustic Prediction and Interior Noise Control Technology", California, NASA, p. 70 (2001).

[13] X. Wang, N. Hampl, T. Ahlersmeyer, S. Watkins, S.J. Elliott, D.C. Baillie, D. Vigé, B. Reff, and G.M. Goetchius, "Vehicle Noise and Vibration Refinement", Woodhead Publishing Limited (2010).

[14] F.A. Everest and K.C Pohlmann, "Absorption, Master Handbook of Acoustics". 5 ed: McGraw-Hill, pp. 179-221 (2009).

[15] W. Fung and M. Hardcastle, "Textiles in Automotive Engineering", Cambridge: Woodhead Publishing Limited (2001). 
[16] D.V. Parikh, T.A. Calamari and J.C. Myatt, "Performance of Nonwoven Cellulosic Composites for Automotive Interiors", International Nonwovens Journal, 9(2), pp. 83-85 (2000).

[17] S. Donders, "Computer-Aided Engineering Methodologies For Robust Automotive NVH Design", Thesis, Katholieke Universiteit Leuven (2008).

[18] C.B. Nel, "Optimisation of Engine Mount Systems for Front-Wheel-Drive Vehicles", Thesis, Technikon Pretoria, Pretoria (1997).

[19] S.S. Rao, "Mechanical Vibrations", 5th ed., Singapore, Pearson (2011).

[20] DYTRAN, "Accelerometer Mounting Considerations", Dytran Instruments (2013).

[21] J. Malchaire, "Occupational Exposure to Noise: Evaluation, Prevention and Control", In: B. Goelzer, C.H. Hansen and G.A. Sehrndt., editor. Sound Measuring Instruments, WHO, pp. 125-140 (2001).

[22] Brüel, \& Kjær, "Measuring Sound", Denmark, pp. 45 (1984).

[23] L. Zheng, Z. Fang, Z. Tang, Z. Zhan and J. Fu, "The Design Optimization of Vehicle Interior Noise through Structural Modification and Constrained Layer Damping Treatment", SAE International (2015).

[24] R.K. Dunne, D.A. Desai and R. Sadiku, "A Review of Porous Automotive Sound Absorbers, their Environmental Impact and the Factors that Influence Sound Absorption", Int J Vehicle Noise and Vibration, 13(2), pp.137-163 (2017).

[25] R. Dunne, D. Desai and R. Sadiku, "A Review of the Factors that Influence Sound Absorption and the Available Empirical Models for Fibrous Materials", Acoustics Australia, 45, pp. 453-469 (2017). 
FIGURES AND TABLES

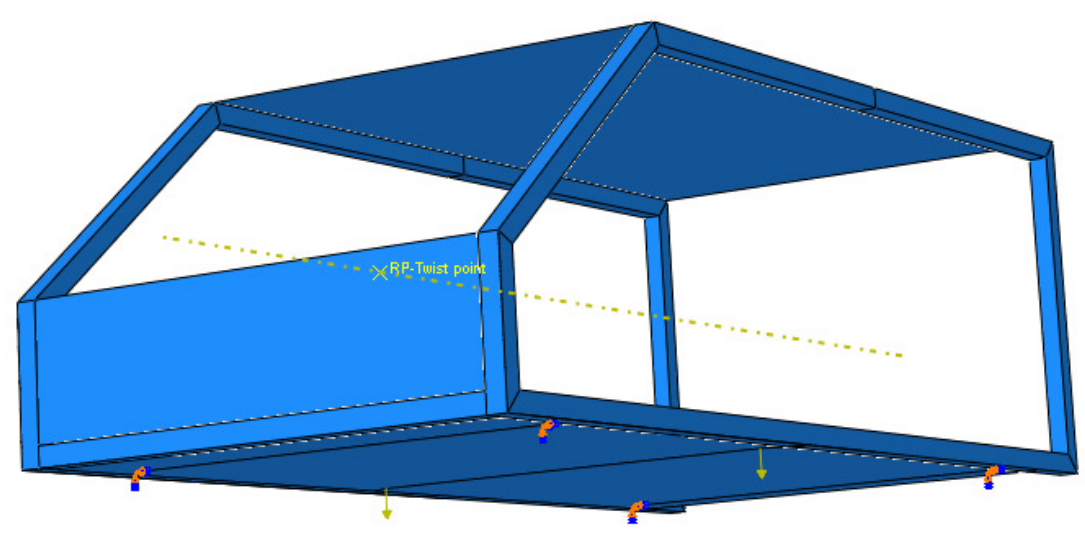

Fig.1. Scaled ABAQUS BIW model

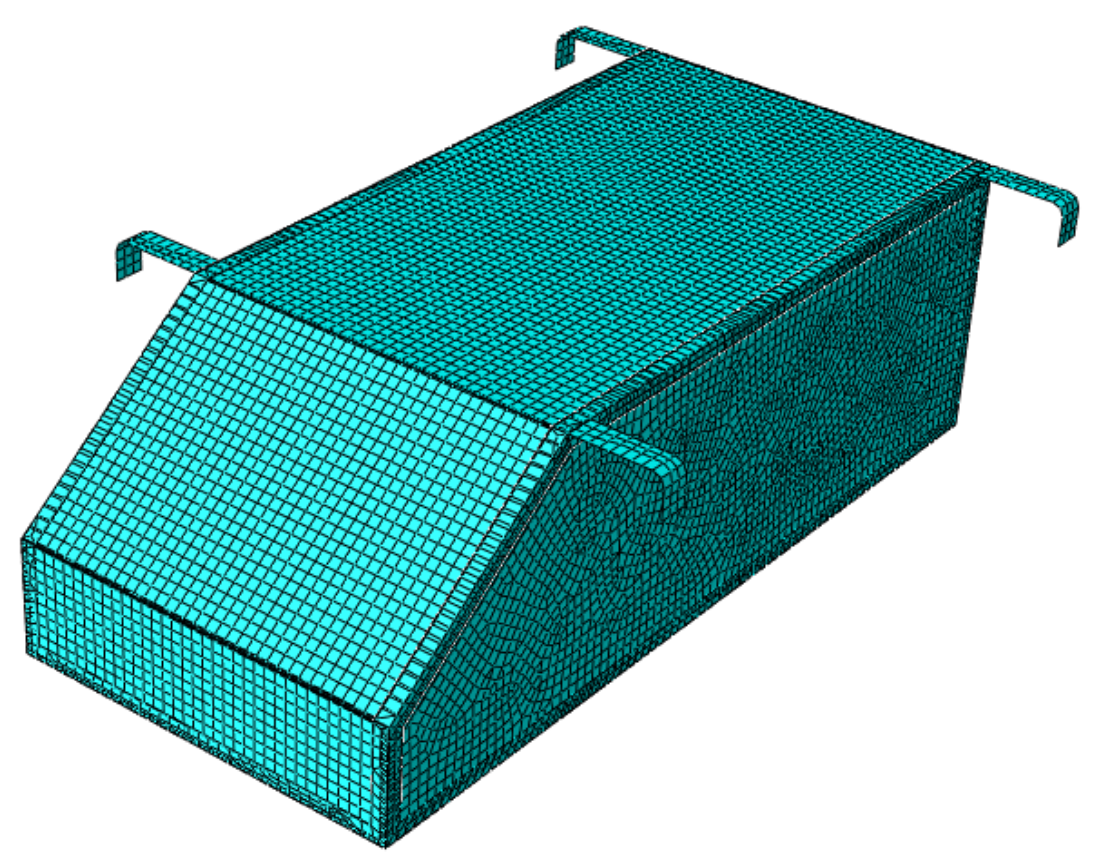

Fig.2. Scaled complete vehicle finite element model 


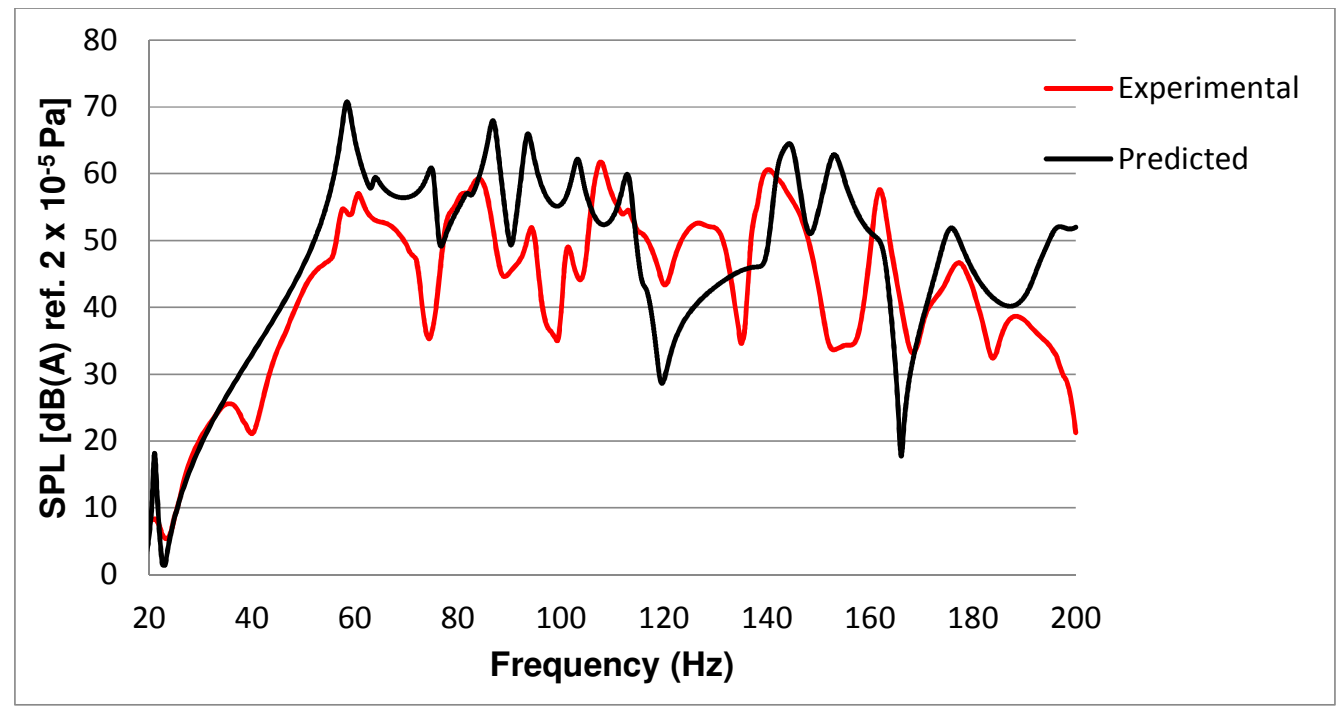

Fig.3. Measured and predicted SPL's at driver's head position with Sisal / Kenaf material model

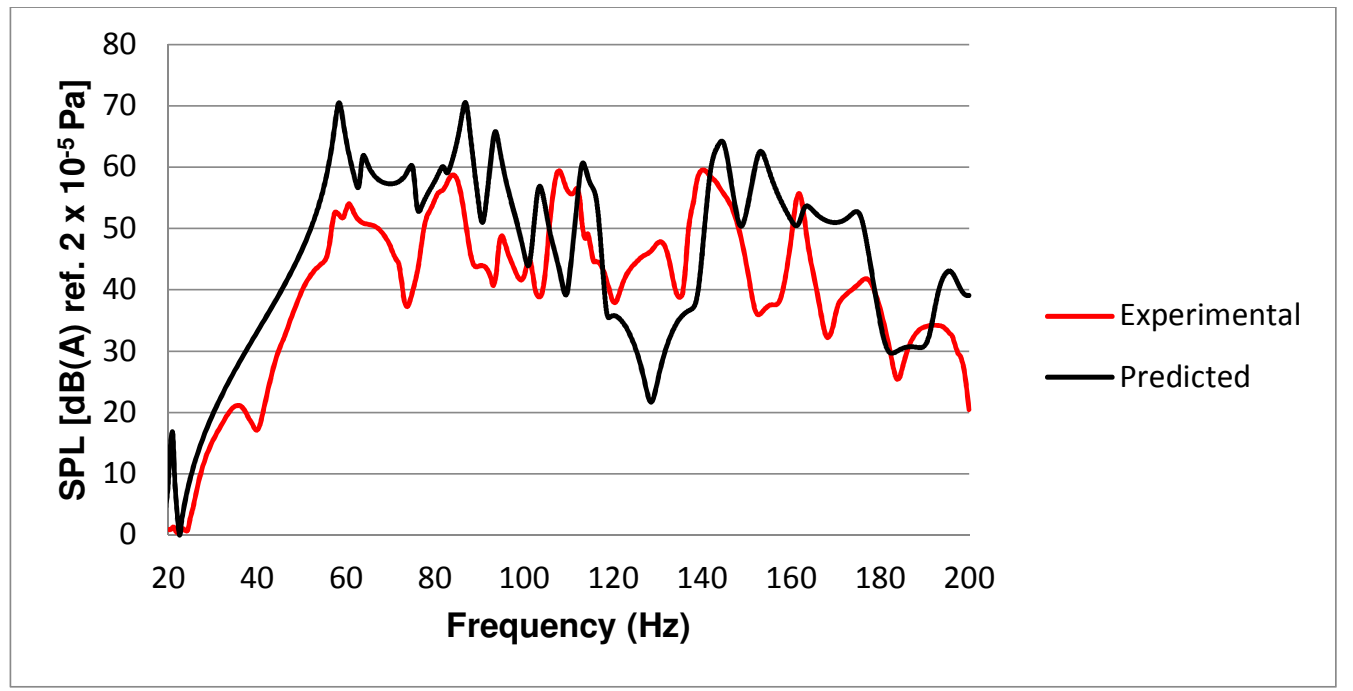

Fig.4. Measured and predicted SPL's at rear passenger's head position with Sisal / Kenaf material model 


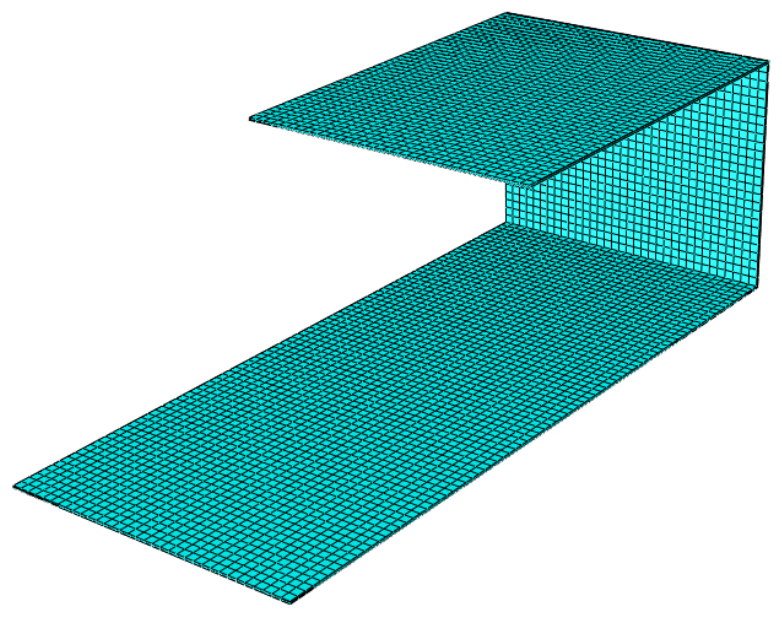

Fig.5. Finite element model of the poroelastic natural fibre composite

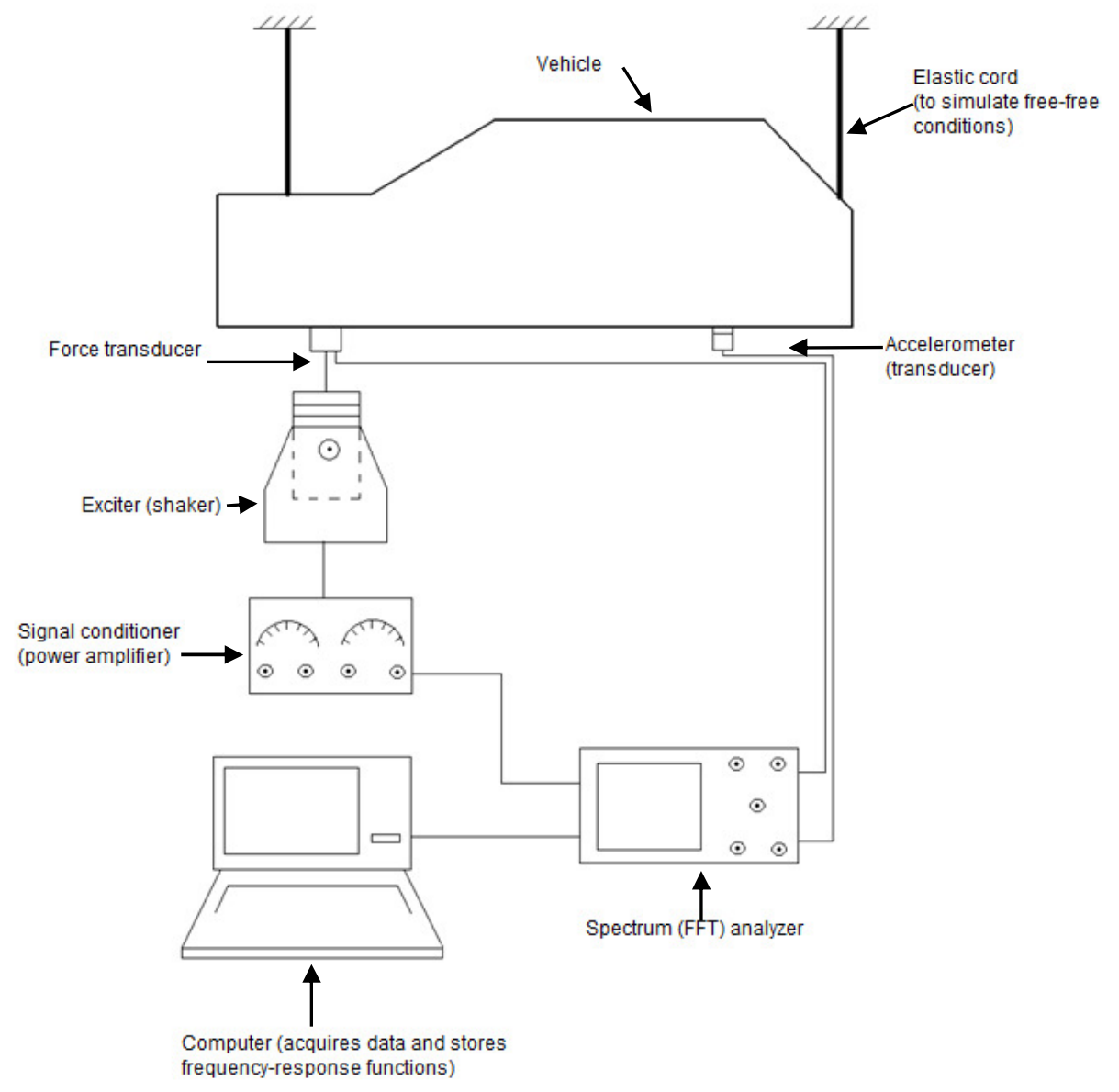

Fig.6. Schematic of experimental test setup 

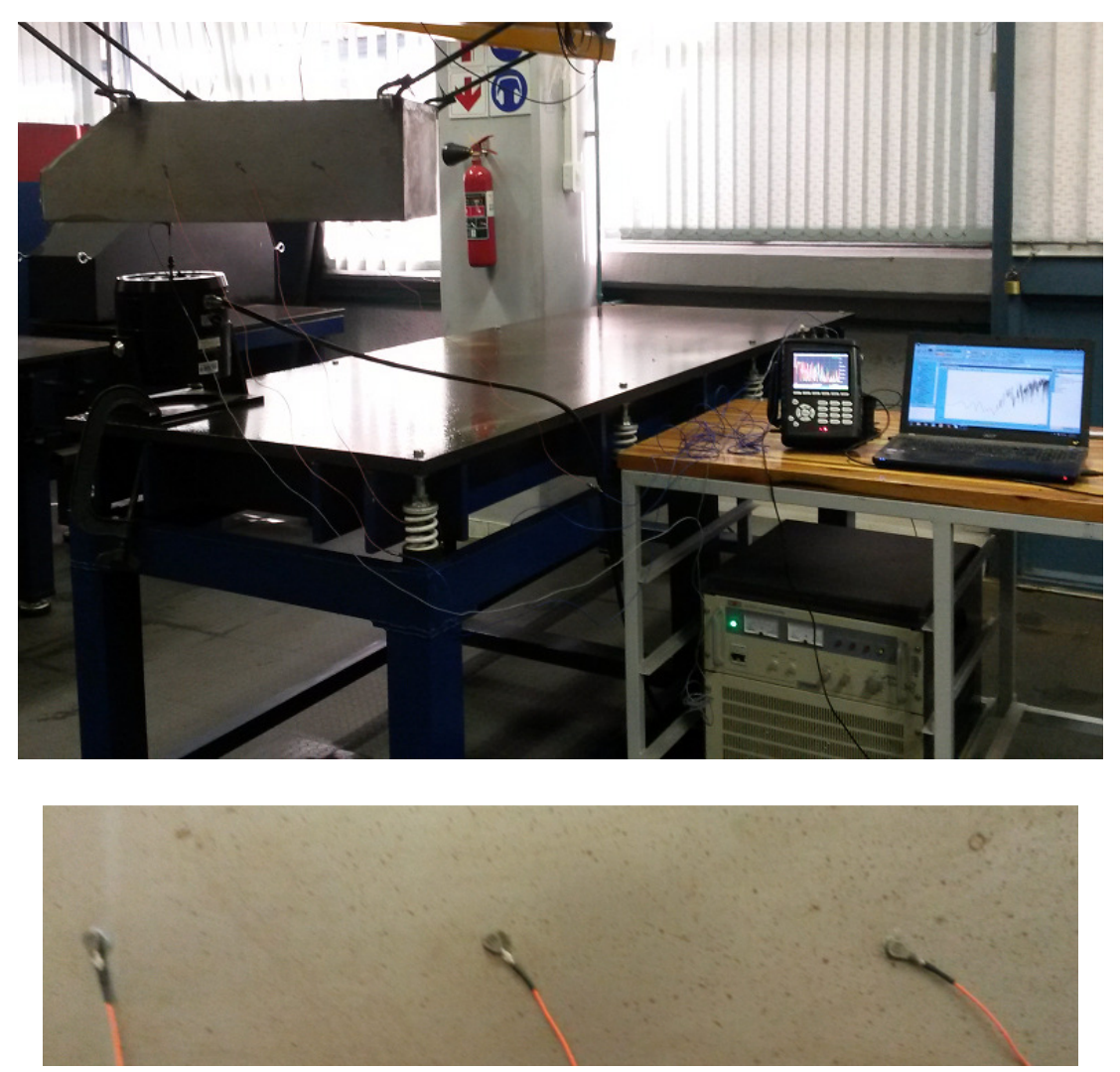

Fig.7. A photograph of constructed model with attached accelerometers

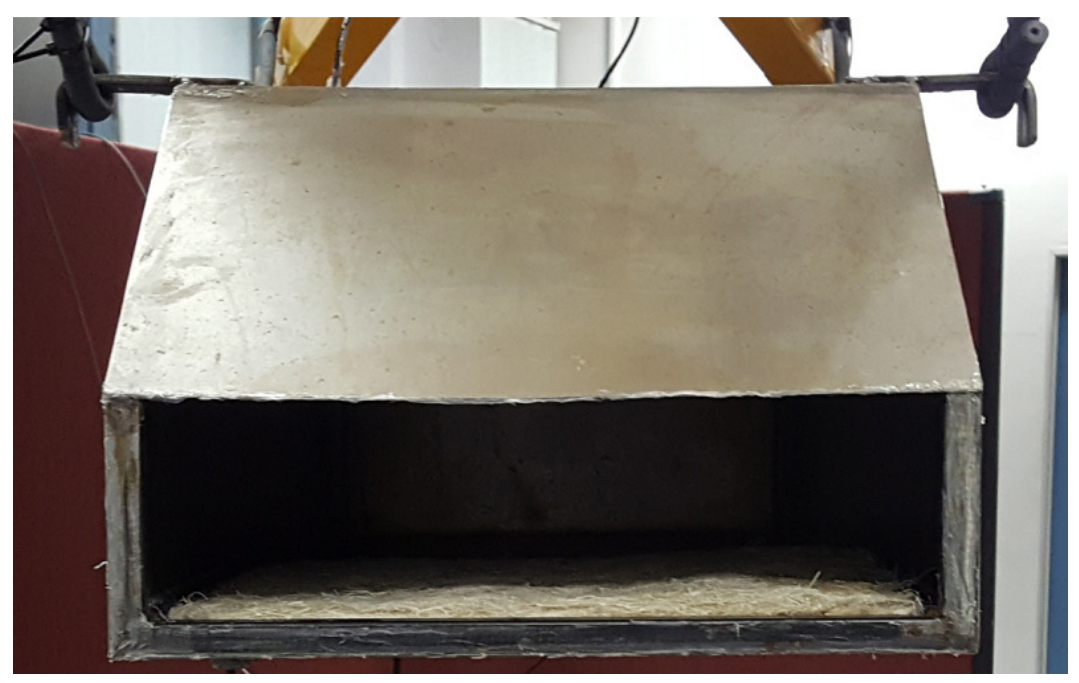

Fig.8. A photograph of constructed model with natural fibre composite (front view) 

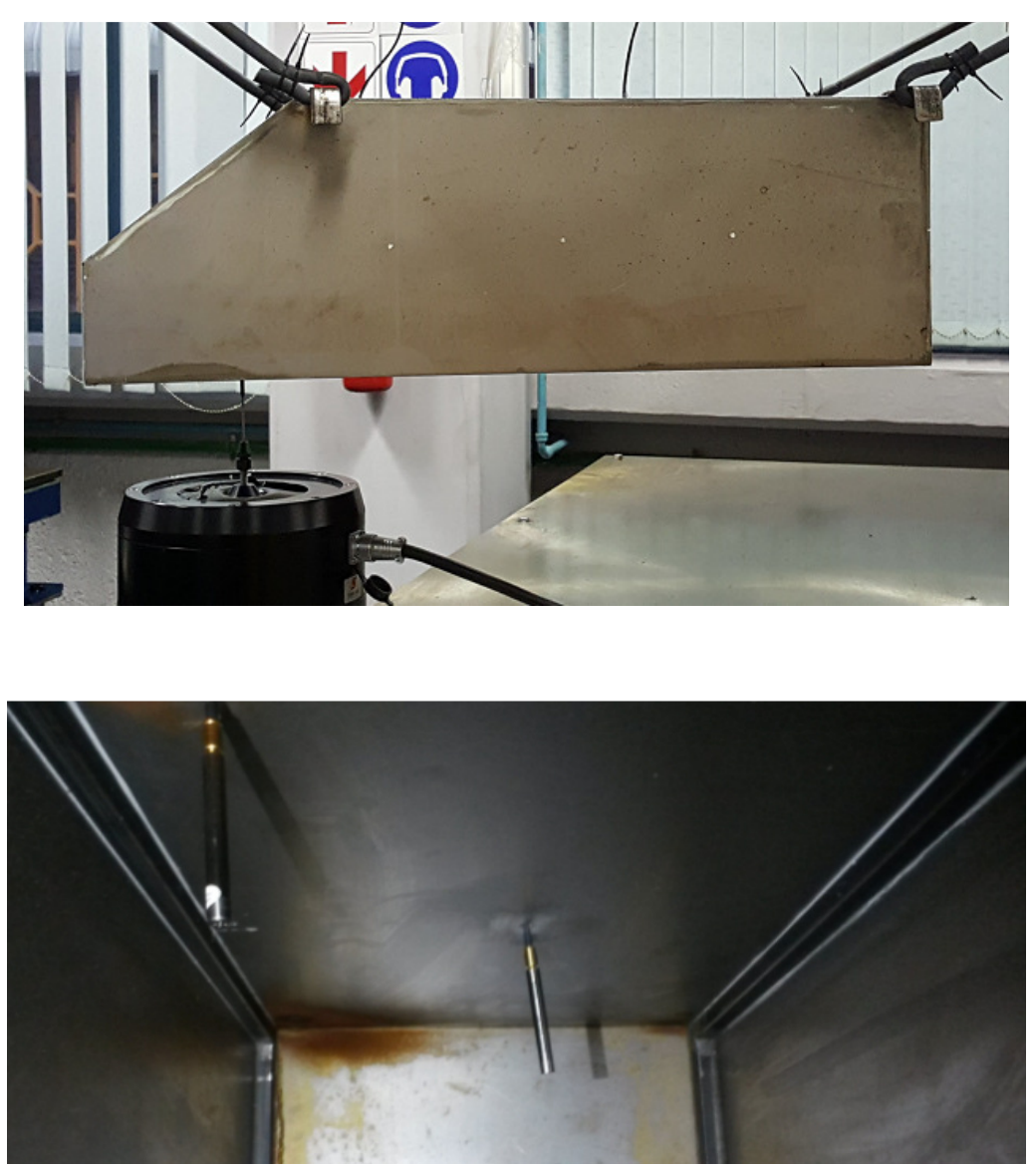

Fig.9. A photograph of constructed cabin with attached microphones.

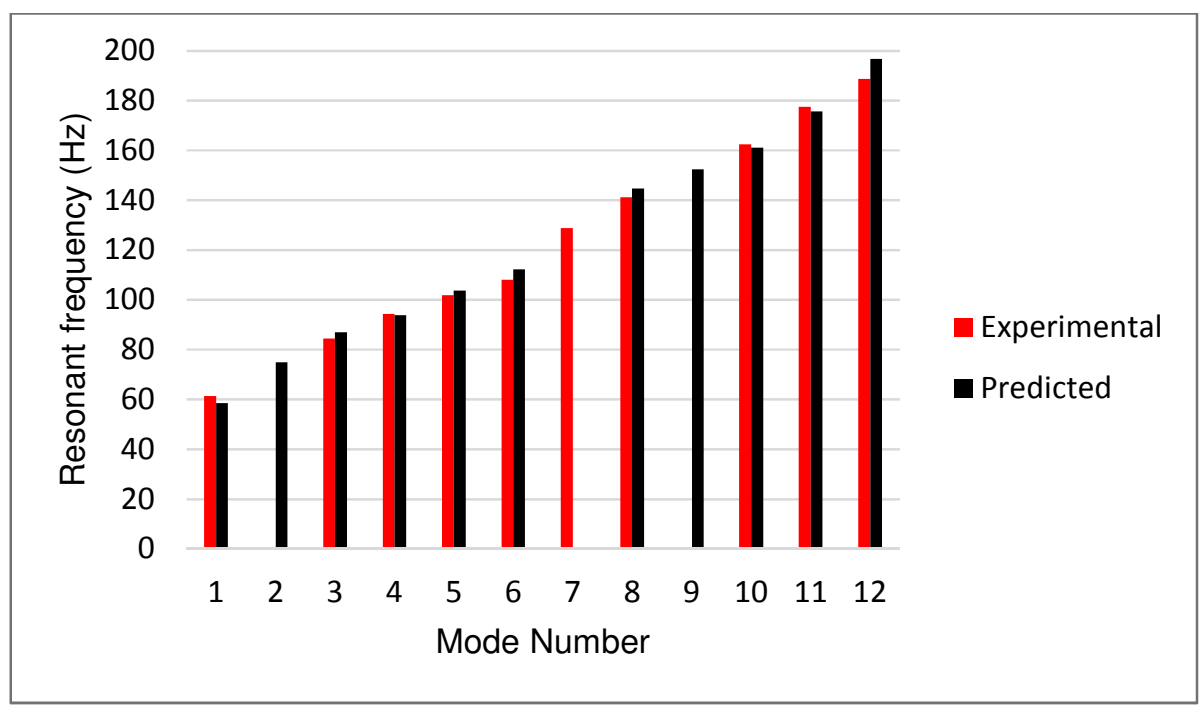

Fig.10. Relation between experimental and predicted resonant frequencies 


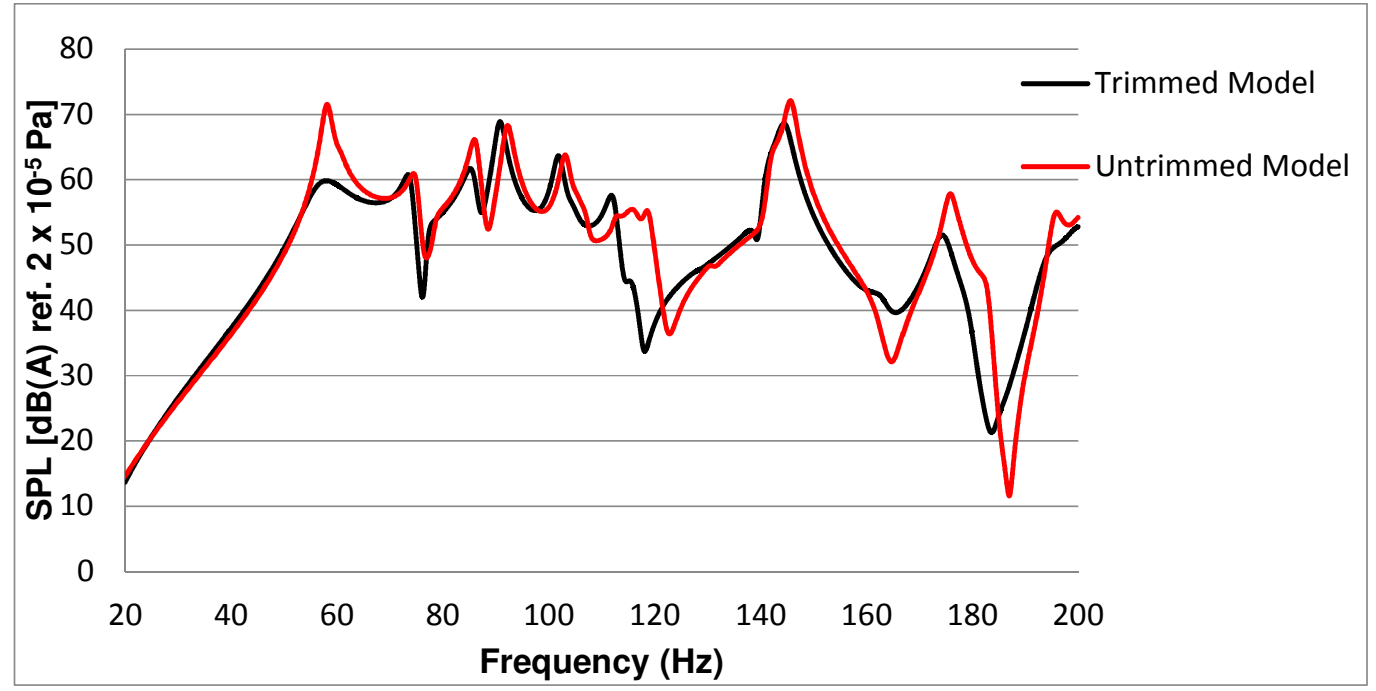

Fig.11. Predicted SPL's of trimmed and untrimmed model at driver's head position.

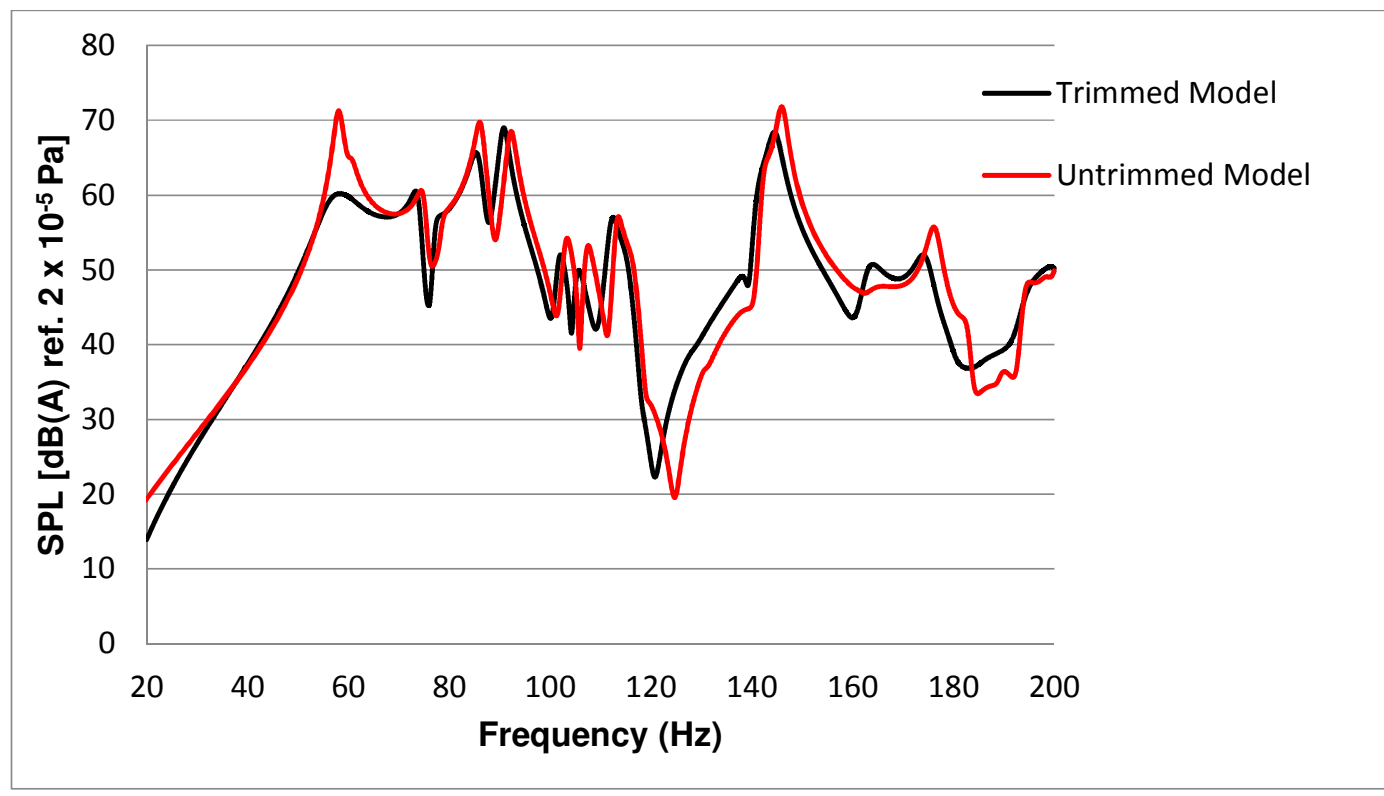

Fig.12. Predicted SPL's of trimmed and untrimmed model at rear passenger's head position 
Table 1. Acquired material properties of $70 \%$ Sisal / $30 \%$ Kenaf fibre ABS composite.

\begin{tabular}{|c|c|c|c|c|c|}
\hline $\begin{array}{c}\text { Bulk } \\
\text { Density } \\
\mathbf{k g} / \boldsymbol{m}^{\mathbf{3}}\end{array}$ & $\begin{array}{c}\text { Permeability } \\
\boldsymbol{m s}^{-\mathbf{1}}\end{array}$ & Void Ratio & $\begin{array}{c}\text { Log Bulk } \\
\text { Modulus }\end{array}$ & $\begin{array}{c}\text { Shear } \\
\text { Modulus } \\
\mathbf{M P a}\end{array}$ & $\begin{array}{c}\text { Tensile } \\
\text { Strength } \\
\boldsymbol{k P a}\end{array}$ \\
\hline 117.242 & 0.00307 & 9.362 & 0.605 & 3.572 & 305.935 \\
\hline
\end{tabular}

Table 2. Difference in trimmed and untrimmed SPL's with reference to Figs. 11 and 12.

\begin{tabular}{|c|c|c|c|}
\hline \multicolumn{5}{|l|}{ Front head position } & Untrimmed $\boldsymbol{d B}(\mathbf{A})$ & Trimmed $\boldsymbol{d B}(\mathbf{A})$ & Difference in $\boldsymbol{d B}(\mathbf{A})$ \\
\hline Mode & 71.45 & 60.36 & 11.09 \\
\hline 1 & 66.14 & 61.65 & 4.49 \\
\hline 3 & 72.11 & 67.35 & 4.76 \\
\hline 7 & 57.8 & 51.42 & 6.38 \\
\hline 8 & 55 & 50.32 & 4.68 \\
\hline 9 & \multicolumn{3}{|c|}{} \\
\hline Rear head position & Untrimmed $\boldsymbol{d B}(\mathbf{A})$ & Trimmed $\boldsymbol{d B}(\mathbf{A})$ & Difference in $\boldsymbol{d B}(\mathbf{A})$ \\
\hline Mode & 71.24 & 59.94 & 11.3 \\
\hline 1 & 69.35 & 64.98 & 4.37 \\
\hline 3 & 57 & 55.22 & 1.78 \\
\hline 7 & 71.82 & 68.2 & 3.62 \\
\hline 8 & 55.7 & 51.22 & 4.48 \\
\hline 9 & & &
\end{tabular}

